\title{
Breast cancer: are long-term and intermittent endocrine therapies equally effective?
}

\author{
Jutta Engel ${ }^{1} \cdot$ Gabriele Schubert-Fritschle $^{1} \cdot$ Rebecca Emeny $^{2} \cdot$ Dieter Hölzel $^{1}[$
}

Received: 3 April 2020 / Accepted: 14 May 2020 / Published online: 29 May 2020

(c) The Author(s) 2020

\begin{abstract}
Purpose In breast cancer (BC), the duration of endocrine adjuvant therapies (AT) has been extended continuously up to 10 years. We present an alternative explanation for the effect, which could enable shorter treatments.

Method The relevant literature on chemoprevention and (neo-)adjuvant therapy was reviewed. Data for initiation and growth of primary and contralateral BCs and their metastases (MET) were considered. Also, population-based data from the Munich Cancer Registry for MET-free survival, time trends of MET patterns, and survival achieved by improved ATs are used to estimate all events in the long-term follow-up.

Results Extended ATs (EAT) that continue after 1, 2, or 5 years reduce mortality only slightly. The effect is delayed, occurring more than 5 years after extension. EATs does not affect the prognosis of 1 stBCs, they preventively eradicate contralateral 2ndBCs and thus their future life-threatening METs. Because chemoprevention can eradicate BCs from the smallest clusters to almost detectable BCs, ATs can be temporarily suspended without imposing harm. Results equal to EATs can be achieved by short-term ATs of the 1stBC and by repeated neo-ATs targeted at the indefinitely developing 2ndBCs. Considering this potential in de-escalation, a 70-80\% reduction of overtreatment seems possible.

Conclusion Knowledge of initiation and growth of tumors with known effects of neo-ATs suggest that intermittent endocrine ATs may achieve the same results as EATs but with improved quality of life and survival because of fewer side effects and better compliance. The challenge for developments of repeated ATs becomes: how short is short enough.
\end{abstract}

Keywords Breast cancer $\cdot$ Metastasis $\cdot$ Long-term endocrine adjuvant therapy $\cdot$ Over-treatment $\cdot$ Survival

\begin{tabular}{|c|c|}
\hline Abbreviatio & \\
\hline (E)AT & (Extended) adjuvant endocrine therapy \\
\hline (1st/2nd) BC & (First/second) breast cancer \\
\hline MET & Metastasis \\
\hline $\mathrm{TC}$ & Tumor cell \\
\hline VD & Volume doubling \\
\hline
\end{tabular}

Dieter Hölzel

hoe@ibe.med.uni-muenchen.de

1 Munich Cancer Registry (MCR), Institute for Medical Information Processing, Biometry and Epidemiology (IBE), Ludwig-Maximilians-University (LMU), 81377 Munich, Germany

2 The Dartmouth Institute for Health Policy and Clinical Practice, Geisel School of Medicine at Dartmouth, Lebanon, NH 03756, USA

\section{Introduction}

The goal of adjuvant therapies (ATs) is to reduce BC mortality. This is only possible by reducing life-threatening metastases (METs), either by eradication or prevention. ATs have improved the prognosis of BC significantly over the past decades. 15-year tumor-specific survival should now exceed $80 \%$ in comparison to 55\% decades ago (Hölzel et al. 2017; Noone et al.; Welch et al. 2016). Endocrine ATs with tamoxifen and aromatase inhibitors showed an additional delayed survival benefit of less than $3 \%$ when extended to 5,10 and more years (Bartlett et al. 2019; Davies et al. 2013; Goss et al. 2016; Gray 2013). This broad range of treatments has been considered extended AT (EAT) and guidelines already recommend 10 years of ongoing ATs (Burstein et al. 2019; Cardoso et al. 2019). Analysis of population-based data from the Munich Cancer Registry (Munich Cancer Registry) with long-term follow-up and changing MET patterns (Hölzel et al. 2017) have revealed principles of MET that suggest a critical review of EATs. The aim of this review is to explain 
the proven benefit of EAT and to discuss functionally equivalent AT strategies of intermittent ATs based on principles of tumor biology and empirical knowledge.

\section{Methods}

The article is based on a review of pertinent randomized controlled trials and meta-analysis of BC mortality to be considered for explaining the effect of EATs. First, the effects of neoadjuvant and ATs are considered. In ATs, we focus on the important distinction between the effects of delayed AT versus EATs. The second major focus concerns the initiation and growth of BCs and their METs. Chemoprevention is particularly relevant, because it provides data for the risk reduction of first and second $\mathrm{BCs}(1 \mathrm{stBC} / 2 \mathrm{ndBC})$ and for their growth duration.

Third, population-based data of the Munich Cancer Registry (MCR) are taken into account, whose versatile analysis ultimately led to the results presented herein, including the generation of the hypothesis that EAT maybe considered as overtreatment. We consider MET-free survival with a follow-up of 20 years and more, as well as time trends of the MET pattern and survival achieved by improved ATs. Analyses of MCR data have revealed relevant principles of MET and their treatment. Known data on the incidence of $1 \mathrm{st} / 2 \mathrm{ndBCs}$ are also considered. These data and combinations of the effects of short-, long-term, delayed and preventive neo-ATs provide an explanation for the remarkable delay in effect of EATs.

\section{Results}

\section{Growth and initiation of breast cancer}

Growing BCs can be described in four ways, by the number of tumor cells (TC), the duration of growth, the diameter of BCs and a molecular timeframe which distinguish phases with relevant mutations that disseminated TCs can inherit (Fig. 1a) (Yates et al. 2017). The growth of BCs has been estimated from mammography screening. For 60-70 year old patients, the median volume doubling (VD) time for tumor growth from 10 to $20 \mathrm{~mm}$ is 143 days, with a variation from 65 to 308 days for the 25th and 75th percentile (WeedonFekjaer et al. 2008). Applying these percentiles, a pT1a$\mathrm{BC}$ of $3 \mathrm{~mm}$ diameter would grow to a pT2-BC of $28 \mathrm{~mm}$ (the average of pT2) after 9.6 VDs within 1.7/3.8/8.2 years, respectively. The biennial screening with $20-25 \%$ interval cases confirms the variability.

If we assume a constant growth with these three percentiles from the first TC, then a pT1c-BC will be reached after approximately 31.5 VDs or after 5.6/12.3/26.6 years. Population-based estimates reveal only about $7 \%$ fast-growing triple negative $\mathrm{BCs}$, that is, even the growth rate of hormone receptor positive $(\mathrm{HR}+) \mathrm{BCs}$ varies by a factor of 10 and more. These growth rates are plausible, because in
Fig. 1 Initiation of metastases. Growing BCs (a) can initiate metastases (c, d), true local recurrences (e), positive lymph nodes (f) over time through dissemination of heterogeneous tumor cells (b). If not diagnosed synchronously, these secondary foci as well as $2 \mathrm{ndBC}(\mathrm{g})$ are already prevalent at diagnosis (h) and can be affected by ATs which are short compared to the growth duration of METs. TC tumor cell, $T D$ tumor diameter, $M E T$ metastasis, $A T$ adjuvant therapy, $B C S$ breast conserving surgery

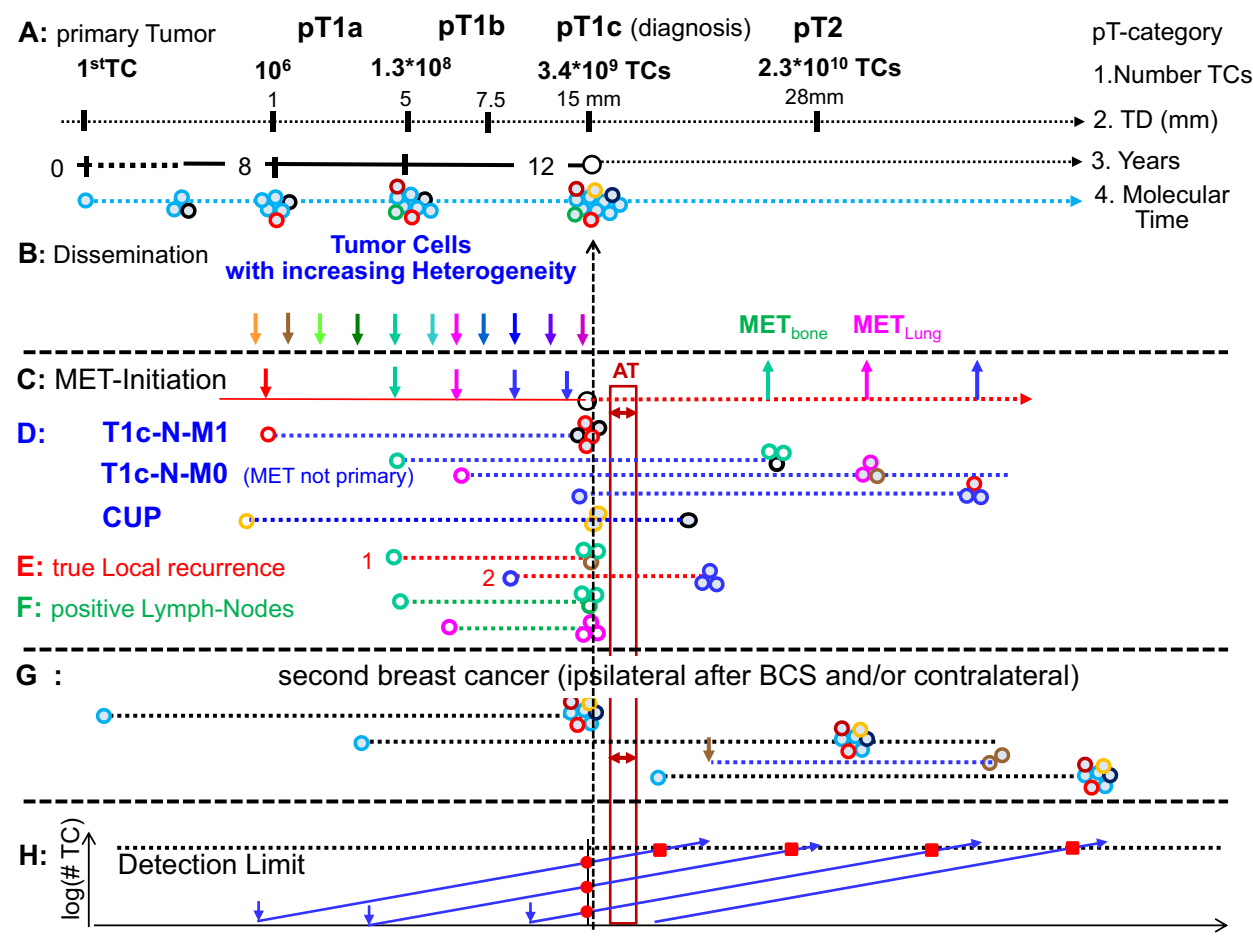




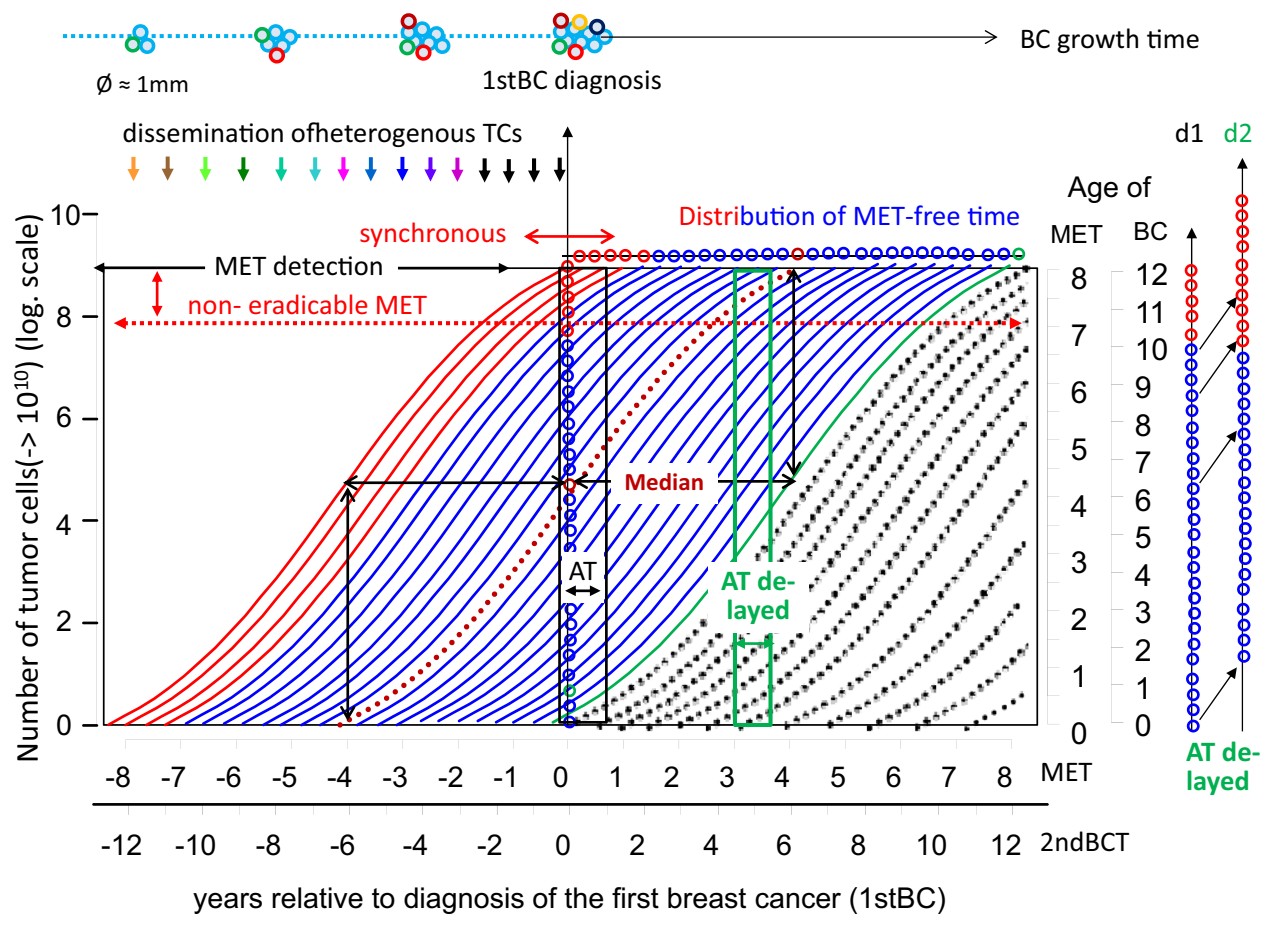

Fig. 2 Growth trajectories (GT) for BCs and METs. The time scales (median growth time 8 years for METs and 12 years for 2ndBCs) distinguishes times before and after BC diagnosis. Early initiated, synchronously diagnosed and no longer eradicable METs (red GTs), a median GT (brown dotted) and a late-initiated GT (green) are outlined. The age scale represents the age of prevalent METs at BC diagnosis. In addition, age distributions of METs are outlined in patient

prevention trials the incidence reduction persists for at least 15 years after the end of chemoprevention (Cuzick et al. 2015). Therefore, contralateral $2 \mathrm{ndBCs}$ are already prevalent and may be diagnosed within the next 12.3 years after the removal of the $1 \mathrm{stBC}, 2 \%$ synchronous BCs are the first of them. 2ndBCs that appear after 12.3 years are increasingly initiated year after year following the removal of the $1 \mathrm{stBC}$ (Fig. 1g).

\section{Initiation and growth of MET}

The initiation of METs can be read from survival data. According to our data, the tumor-specific 15-year survival of pT1- and pT2-BCs was at $76 \%$ and $53 \%$ in the period 1988-1997. Therefore, when a pT1c-tumor with a diameter of $15 \mathrm{~mm}$ is reached, at least $24 \%$ already had early initiated METs. However, more than 23\% additional METs will be initiated while growing up to $28 \mathrm{~mm}$, which totals $47 \%$ METs that could not be eradicated by former use of ATs. A Gompertz function can be fitted for the S-shaped relation of tumor diameter and tumor-specific mortality, which results cohorts with immediate or delayed onset of ATs. Also outlined is the short duration of today's ATs (black and green rectangle) compared to the growth period of METs. With the time scale for 2ndBCs, GTs are to be interpreted analogously. The dotted GTs represents 2ndBCs initiated after BC diagnosis ( $B C$ breast cancer, $M E T$ metastasis, $A T$ adjuvant therapy, $T C$ tumor cell)

as the complement to $100 \%$ in the following: relative 15 -year survival $(\%)=100-58.39 \times \exp (-4.46 \times \exp (-0.071 \times$ TD)) for tumor diameter (TD) $\leq 90 \mathrm{~mm}$ (Engel et al. 2019). With each additional millimeter, a growing $\mathrm{BC}$ initiates

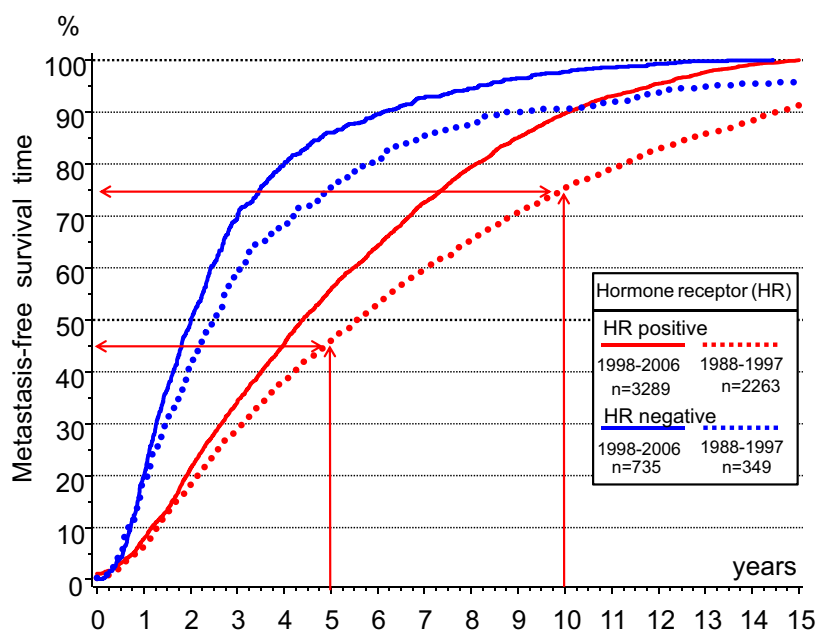

Fig. 3 Distribution functions of MET-free intervals. The dependency on hormone receptor status from BCs and on time periods with different follow-up is to be considered ( $n=6636$ of T-N-M0 BCs) 
0-1.5\% additional METs which are diagnosed in a median time span of 28 months (Munich Cancer Registry) before death.

Initiation, growth and treatment effects can be explained by growth trajectories, with a log scale for the number of TCs in relation to time (Fig. 2). Two growth sections need to be distinguished; before and after the diagnosis of a BC. METs of primary advanced BCs have grown after initiation parallel to the BC and are diagnosed simultaneously. In primary M0 findings, the last MET initiations take place shortly before the BC removal. The sum of growth times before and after diagnosis are the same as for a median MET initiation. The distribution function of the MET-free survival time (Fig. 3) results in a median time of about 4 years. Taken together, a MET should grow a median of 8 years before its detection, or 93 days for one VD (Hölzel et al. 2010). This is long compared to the few months of AT regimes which act on MET (Fig. 2).

This MET process is correct as long as there is no initiation of METs after R0 resections (DeMichele et al. 2017). But it is a contradiction to the dormancy hypothesis, according to which METs can be initiated with time delays. But this explanation is not plausible. If circulating or dormant TCs could initiate delayed METs, then the shortening of each AT from years to a few months would have shown disadvantages, but until today, none have been reported. In addition, a long delay in initiated METs would have to be more often fast-growing triple negative tumors (Fig. 3). That is also not true, and therefore, TCs most likely do not cause clinically relevant METs after BC removal.

\section{Effects of neoadjuvant and adjuvant therapies}

Despite successful ATs, the survival after MET has not greatly improved and has now a median of about 28 months. Improving survival means successfully targeting occult METs. METs of HR + BCs grow slower than HR-BCs. About 50/90\% of MET of HR + BCs occur within a METfree period of 5.6/15 years in comparison to 2.5/8.5 years for HR- BCs (Fig. 3). AT trials show no effect in the first few months. A possible reason is the exclusion of advanced BCs by staging, so that the remaining MET need more time up to detection (Fig. 2). Since diagnosed METs remain ineradicable, this also applies to these larger undetected METs which occur in the first few months following BC diagnosis. Only then, after about 6 months or 1-2 VDs of METs do METfree survival curves open like scissors. The mortality reduction is delayed for another 28 months. Even if treatments lasted only 1 year, there was a reduction in METs after 5 and 10 years (Early Breast Cancer Trialists' Collaborative Group 1992).

The effectiveness of ATs is also manifested in delayed onset. Even a short delay of AT by 2 months after surgery leads to worse outcomes, because some prevalent METs grow to a no longer eradicable size in that short period of time, which is otherwise prevented by ATs by the annihilation of METs within one VD (Gagliato et al. 2014; Yung et al. 2020). The distribution d1, outlined in Fig. 2 represents a cohort of patients in whom METs were continuously initiated. At BC diagnosis, there are occult METs, from smallest clusters to almost detectable foci. The altered age structure of occult METs when initiations of AT are delayed describes distribution $\mathrm{d} 2$ with less tiny and more already detected METs.

Two studies show the effect of delayed starts of tamoxifen treatment after 2-5 years of tumor-free survival (Delozier et al. 2000; Veronesi et al. 2010). In a cohort of patients, when ATs start after a 5 year delay, almost $50 \%$ of all patients who are expected to develop METs during the first 5 years are already excluded (Fig. 3). The effect of these delayed ATs on METs which are still occult after 5 years is similar to the scissors-like opening of immediately initiated ATs for all METs. However, there are no longer small TC clusters, because they have grown in size during the time of the treatment delay. This comparability confirms that occult METs of various ages were eradicated very quickly by ATs until they reach a no longer eradicable size.

\section{Effects of extended adjuvant endocrine therapies}

Studies investigating EATs up to 10 years (Bartlett et al. 2019; Davies et al. 2013; Goss et al. 2016; Mamounas et al. 2019) showed no survival effect for 5 years. This implies that EATs have no effect on METs of $1 \mathrm{stBCs}$, although $55 \%$ or $25 \%$ of METs will occur after 5 or 10 years, respectively (Fig. 3). The ineffectiveness is plausible if there are resistant METs. But there is no tumor biology model so far that explains delayed eradication of METs. That is why the question arises, which effect will be achieved by the extension of ATs from 1 or 2 years to 5 years. 1-2 years of ATs was shown to improve survival even after 10 years (Early Breast Cancer Trialists' Collaborative Group 1998; Ekholm et al. 2016). Comparisons of 2 versus 5 years of tamoxifen(Swedish Breast Cancer Cooperative Group 1996) and also placebo-controlled comparisons after 2 years of AT (Rutqvist and Johansson 2007) show that survival does not change as was also the case of immediate receipt of AT after 1 year. Additionally, the extension of ATs after 1 or 2 years have a delayed effect as in the 5/10-year comparisons, although 95 or $85 \%$ of METs occur later (Fig. 3) (Bartlett et al. 2019; Davies et al. 2013). A delay in MET eradication of more than 5 years is also evident in the 5-year placebo comparison of tamoxifen (Early Breast Cancer Trialists' Collaborative Group 2011). Therefore, the phase of more than 5 years of ineffectiveness of EATs is very likely to be constant and transferable with EATs used after one year. 


\section{Effects of successful adjuvant therapies over decades}

Changes in MET patterns observed in the MCR over decades of successful AT use is an important aspect (Hölzel et al. 2017). In comparison to MET patterns of earlier decades and today's primary advanced BCs, bone and lung METs have been reduced by about $50 \%$ and $30 \%$, respectively. METs are destroyed proportionally from the smallest cluster to a non-eradicable MET size. Alternatively, a clinically relevant reduction of liver or CNS METs is not recognizable. Therefore, the inability of ATs to act equivocally in any micro-environment of MET is one limitation of today's ATs. Another limitation is that even the smallest clusters show acquired resistance, because even very late bone or lung MET occur which would have to have been very small during AT. Of particular note is that the historical success of ATs is almost independent of BC size. It is achieved in BCs with few positive lymph nodes, the proportion of which is largely the same for larger BCs (Engel et al. 2019; Welch et al. 2016).

\section{Effects of chemoprevention}

Information about the growth period of BCs demonstrates that $1 \mathrm{st} / 2 \mathrm{ndBCs}$ are prevalent over many years. Therefore, chemoprevention acts as a neo-AT for already initiated or newly during prevention initiated BCs. Since the effect of prevention already occurs within a few months (Cuzick et al. 2010) and continues even after the completion in the 5th year without risk change for more than 10 years, prevalent $\mathrm{BCs}$ of all sizes are most likely eradicated in 1-2 VDs of BCs, as are METs (Cuzick et al. 2015). This raises the important question of the necessary duration and the rationale of an effective prevention. Regardless of the duration, after the end of any prevention, new BCs develop because the risk of BCs continues throughout life (Fig. 2).

\section{Discussion}

Three types of results have been considered: initiation and growth of BCs and METs with their VD times, the effects of randomized trials on adjuvant, neoadjuvant, extended and preventive therapies, and population-based data on METfree survival along with changing MET patterns. This available evidence results in the following six statements:

First, if the start of ATs is delayed by even a few months or 1,2 or 5 years, they will act on the then still prevalent occult METs in the same way they would if immediately starting ATs (Delozier et al. 2000; Veronesi et al. 2010). But if ATs are prolonged after 1, 2, 5 or 10 years, no comparable short-term effect on occult METs is achieved, but rather only a delayed effect after 5 and more years occurs.

If EATs cannot reduce the 30\% MET from 5 to 10 years, then this should also true for the $25 \%$ METs from the 10th year. It follows that EAT does not reduce METs of the 1stBC (Fig. 3).

Second, the worse outcome after a short delay of ATs and the 5-year of ineffectiveness when AT is prolonged means, that eradicable METs are quickly destroyed and noneradicable METs are resistant. The studies show that METs decrease in the first year, mortality delayed by MET related survival only in the third year. BCs and METs are destroyed shortly after the respective 1-2 VDs. This evidence can already be read from the studies of the 1980s with tamoxifen 1-2 years (Early Breast Cancer Trialists' Collaborative Group 1992, 1998).

Third, EATs also act as neo-ATs on occult 2ndBCs. Three effects are to be distinguished. Most importantly, about 50\% of $2 \mathrm{ndBCs}$ can be eradicated along with their existent and/or future METs (Blok et al. 2018; Early Breast Cancer Trialists' Collaborative Group 1998; Goss et al. 2016). As with neoATs, only METs of 2ndBCs can be eradicated and thirdly, only the $2 \mathrm{ndBC}$ is eradicated. Resistant METs are then most commonly assigned to the $1 \mathrm{stBC}$, but they are cancer of unknown, or more precisely, of already eradicated primary tumors.

Fourth, the magnitude of the delayed effect of EATs seems to be the same regardless of whether ATs are extended

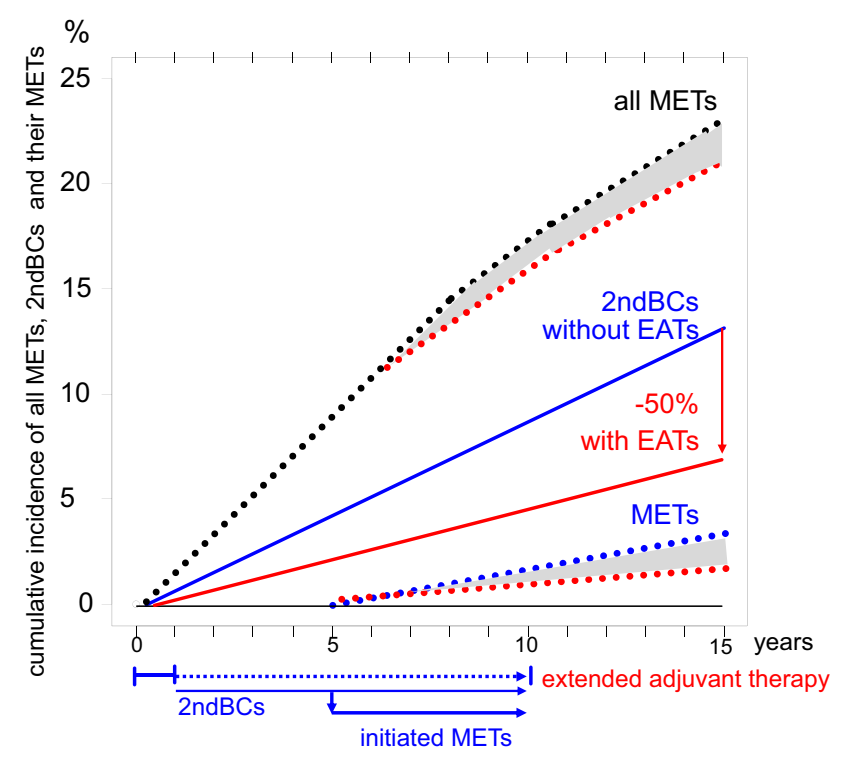

Fig. 4 Effects of EATs by reducing METs from 2ndBCs. The cumulative incidence of METs (black dotted line) is caused by $1 \mathrm{stBCs}$ and 2 ndBCs. If 2 ndBCs (blue line) are reduced by $50 \%$ by EATs (red line), then their METs are also prevented (grey shaded area). Approximately, 4-5 years after the onset of EATs, the reduction in MET risk could become observable because of the BC's growth variability 
after 1,2, 5 or 10 years and is independent from decreasing hazard rates of METs of 1stBCs (Fig. 3). The reason is the incidence of BCs which does not vary much after the age of 55 years, at which point most BC patients are in long-term follow-up (Munich Cancer Registry; Noone et al.). Constant incidences should also apply to the 2ndBCs. Therefore, the delayed constant effect of EATs is due to the effective neoAT on any subsequent 2ndBCs (Fig. 4).

Fifth, regarding the size of the effect, it should be noted that the risk for $2 \mathrm{ndBCs}$ is greater than that for $1 \mathrm{stBCs}$. This increased risk is a surrogate for inherited mutations (Metcalfe et al. 2004; Michailidou et al. 2017). Just a threefold risk(Chen et al. 1999) results in a cumulative 2 ndBC incidence of approximately $17 \%$ after 15 years. It fits the magnitude of these EATs effects (Fig. 4) (Chaudary et al. 1984; Gierach et al. 2016). Subgroups with classical Gail risk factors also reach such a magnitude for the $1 \mathrm{stBC}$. The MET risk reduction should also be particularly large because the 2ndBCs are not yet detectable and if at all have initiated only small single METs.

Sixth, the duration of inefficiency of EATs is important to be considered. METs and subsequent BC-related death can occur 20 years after primary ATs (Fig. 3) (Pan et al. 2017). Therefore, mortality is dominated in EAT studies by the MET risk of 1stBCs with a delayed small improvement, which is postponed more than 5 years for each successive AT extension. This small EAT effect provides an insight into the tumor process: initiation of BCs up to tumor-related death can occur in HR + BCs within 10 years.

EATs work because they prevent METs from contralateral 2ndBCs. The assessment of the effect is made more difficult when the cumulative incidence of METs or mortality is presented in EAT trials with a time lag up to the prolongation of AT and not from diagnosis (Davies et al. 2013). Figure 4

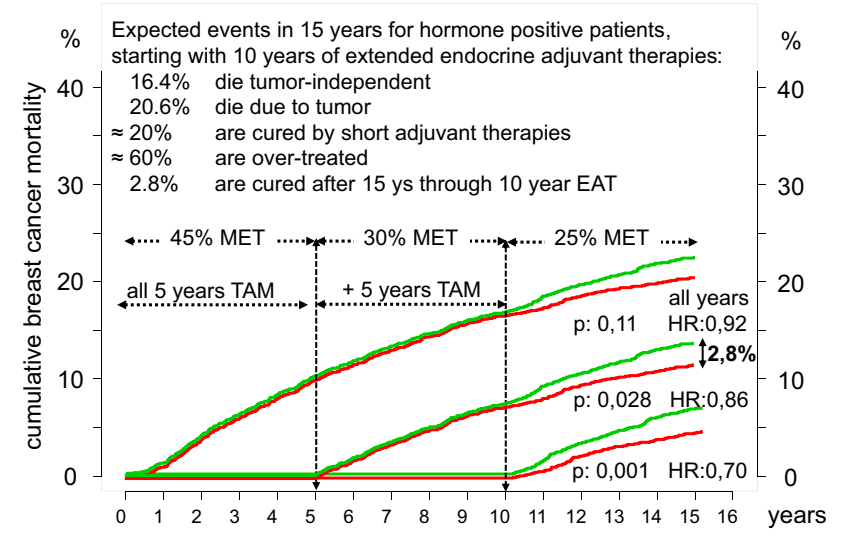

Fig. 5 Simulations with $2 \times 3500$ patients with the expected mortality* and the small effect after 10 years. The survival is shown from the beginning with the common ATs, from randomization of the EAT (red, control green) after 5 years and from 10 years onward. *Mortality was simulated using the distribution in Fig. 3 outlines the current incidence of METs from both $1 \mathrm{stBC}$ and 2ndBC, and the expected effect of an EAT extended from the first year onwards. Consequences of 10 year of EATs show a simulation with today's survival (Fig. 5). The hazard ratio of 0.7 is formally correct from the 10th year on (Davies et al. 2013). But for shared decision making, full information must be provided about all events, in particular because the benefits of 15 years of EATs have already been pointed out (Harbeck et al. 2019). This also includes the many risks of endocrine ATs such as flush, osteoporosis, or fractures and the elaborate efforts to prevent and control side effects. Not even $50 \%$ of patients complete a 5 -year therapy, indicating the burden of EATs.

EATs do not present a new mechanistic principle of action. They act like any AT, but on newly initiated, and eradicable 2 ndBCs, which develop year after year in 1stBC patient cohorts. Because the historical success of AT is independent of tumor size (Engel et al. 2019; Welch et al. 2016), it is not plausible to recommend EAT depending on the risk of recurrence of the 1stBC (Burstein et al. 2019) but rather on the risk and growth rates for 2ndBCs, which should be independent of the prognosis of the $1 \mathrm{stBC}$.

\section{Intermittent endocrine therapies}

The improvement of $2.8 \%$ survival by EATs most likely comes from eradication of $2 \mathrm{ndBCs}$. Immediately initiated ATs also have a chemopreventive effect on prevalent 2ndBCs and, like the METs of the 1stBCs, are quickly destroyed. Extension of ATs cannot improve the prognosis of the 1 st and prevalent 2ndBCs. However, year after year, new 2ndBCs are initiated, which EATs then always act on for all future 2ndBCs. This continuous use of EAT prior to a possible future initiation of $2 \mathrm{ndBC}$ could be considered misuse and overtreatment. This leads us to a new treatment paradigm with intermittent treatments and the question: how short is short enough.

A change of perspective is difficult if randomized trials with extended ATs show improvements in survival. In addition, shorter treatment durations are generally cautiously discussed because hope and interest are more focused on increasing the effect with prolonged therapies (Cameron et al. 2017). In particular, early animal experiments suggested long durations for chemoprevention and ATs, which showed "...that BC development is best inhibited in the constant presence of an anti-oestrogen... and ... reduction in the number and sizes of mammary tumors developing during continuous therapy" (Jordan and Allen 1980). Such a promising possibility to block the carcinogenic process can hamper. Yet in hindsight, the relation of BC and MET growth periods, the length of the therapeutic window and the duration of treatment were not as indisputably available 
then as they are today and were not thoroughly considered in the transfer to humans.

The available evidence suggests that after first ATs that successfully target prevalent METs of the 1stBCs as well as prevalent $2 \mathrm{ndBCs}$, therapy-free intervals should follow before ATs are repeated as neo-AT against newly initiated 2ndBCs. The duration of the break has already been suggested in the ATLAS trial, because almost no METs occur in the first 5 years, a 5-year interval subdivided into 1-1.5year treatment intervals, and 4-3.5-year treatment-free periods. ATs repeated after the break will chemopreventively eradicate $50 \%$ of new initiated BCs. The short treatment is sufficient, because the available endocrine therapies such as those that target e.g. HER2(Cameron et al. 2017) also have fast responses within few VDs times.

Such changes are already suggested. One study is testing an extended intermittent use of AI with 3 month therapy-free intervals each year after completion of 4-6 years of endocrine ATs, a tentative step towards a paradigm shift with treatment de-escalation without disadvantages for patients (Blok et al. 2018; Colleoni et al. 2018; Gnant et al. 2018). Lack of difference between 7 and 10 years of treatments also shows that shorter treatments achieve the same effects. This advancement is also suggested by prevention studies (Cuzick et al. 2015). After 5 years of prevention and an equally long therapy-free interval, a further prevention initiated from the 10th year on repeats the success. This is a convincing intermittent neo-AT of the 1stBC in high-risk patients and is equally applicable to BC patients with high risk of 2ndBCs. Only the intervals have to be adjusted.

The main question about endocrine ATs today is not "how long is long enough"(Smith et al. 2014) but "how short is short enough", an experimental challenge that needs to be supported by further reviews and evaluation of observational data and that could promote logical evidence-based reasoning. The sequential use of aromatase inhibitors and tamoxifen should also be critically examined (Cuzick et al. 2010; Early Breast Cancer Trialists' Collaborative Group 2015).

\section{Conclusion}

The result of EATs is evidence-based but not the interpretation. The eradication of prevalent $2 \mathrm{ndBCs}$ and their future METs cause the effect of EAT and can be achieved with functionally equivalent intermittent ATs. This is logical when cause, effect and growth times of BCs and METs are considered together. Both small clusters as well as large occult foci can already be eradicated by neo-ATs at the beginning of treatment. Therefore, a reduction of treatments of $70-80 \%$ every 5 years seems possible. Shorter treatment durations would significantly reduce the many risks and improve quality of life. Considering the nearly 2 million new HR + BCs worldwide and about 14 million BC patients surviving for more than 10 years, this population burden alone obliges a critical discourse on the rationale of intermittent (neo-) ATs and a reduction of overtreatment. Improved quality of life, a modified chemoprevention for women at high risk for 1stBC and even higher survival rates after the $1 \mathrm{stBC}$ all appear achievable with shorter interval treatments, fewer side effects and thus better compliance.

Acknowledgements Open Access funding provided by Projekt DEAL. We are thankful to all participating doctors and clinicians within our catchment area with meanwhile 4.9 million inhabitants, for having cooperated with us for so many years. In particular, we would like to thank I. Bauerfeind and the members of the working group Breast Cancer of the Munich Tumor Center and many clinicians, whom we have annoyed with our questions on the effects and contradictions of the long-term adjuvant therapies. We also thank our colleagues within the MCR who carefully collected and updated the available data.

\section{Compliance with ethical standards}

Conflict of interest The authors have declared no conflicts of interest.

Open Access This article is licensed under a Creative Commons Attribution 4.0 International License, which permits use, sharing, adaptation, distribution and reproduction in any medium or format, as long as you give appropriate credit to the original author(s) and the source, provide a link to the Creative Commons licence, and indicate if changes were made. The images or other third party material in this article are included in the article's Creative Commons licence, unless indicated otherwise in a credit line to the material. If material is not included in the article's Creative Commons licence and your intended use is not permitted by statutory regulation or exceeds the permitted use, you will need to obtain permission directly from the copyright holder. To view a copy of this licence, visit http://creativecommons.org/licenses/by/4.0/.

\section{References}

Bartlett JMS et al (2019) Breast Cancer Index and prediction of benefit from extended endocrine therapy in breast cancer patients treated in the Adjuvant Tamoxifen-To Offer More? (aTTom) trial. Ann Oncol 30:1776-1783. https://doi.org/10.1093/annonc/mdz289

Blok EJ et al (2018) Optimal duration of extended adjuvant endocrine therapy for early breast cancer; results of the IDEAL trial (BOOG 2006-05). J Natl Cancer Inst. https://doi.org/10.1093/jnci/djx 134

Burstein HJ et al (2019) Adjuvant endocrine therapy for women with hormone receptor-positive breast cancer. ASCO Clin Pract Guidel Focus Update J Clin Oncol 37:423-438. https://doi.org/10.1200/ jco. 18.01160

Cameron D et al (2017) 11 years' follow-up of trastuzumab after adjuvant chemotherapy in HER2-positive early breast cancer: final analysis of the HERceptin Adjuvant (HERA) trial. Lancet 389:1195-1205. https://doi.org/10.1016/s0140-6736(16)32616-2

Cardoso F et al (2019) Early breast cancer: ESMO Clinical Practice Guidelines for diagnosis, treatment and follow-updagger. Ann Oncol 30:1194-1220. https://doi.org/10.1093/annonc/mdz173

Chaudary MA, Millis RR, Hoskins EO, Halder M, Bulbrook RD, Cuzick J, Hayward JL (1984) Bilateral primary breast cancer: a prospective study of disease incidence. Br J Surg 71:711-714 
Chen Y, Thompson W, Semenciw R, Mao Y (1999) Epidemiology of contralateral breast cancer. Cancer Epidemiol Biomark Prev $8: 855-861$

Colleoni M et al (2018) Extended adjuvant intermittent letrozole versus continuous letrozole in postmenopausal women with breast cancer (SOLE): a multicentre, open-label, randomised, phase 3 trial. Lancet Oncol 19:127-138. https://doi.org/10.1016/s1470 $-2045(17) 30715-5$

Cuzick J, Sestak I, Baum M, Buzdar A, Howell A, Dowsett M, Forbes JF (2010) Effect of anastrozole and tamoxifen as adjuvant treatment for early-stage breast cancer: 10-year analysis of the ATAC trial. Lancet Oncol 11:1135-1141. https://doi.org/10.1016/s1470 $-2045(10) 70257-6$

Cuzick J, Sestak I, Cawthorn S, Hamed H, Holli K, Howell A, Forbes JF (2015) Tamoxifen for prevention of breast cancer: extended long-term follow-up of the IBIS-I breast cancer prevention trial. Lancet Oncol 16:67-75. https://doi.org/10.1016/s1470 $-2045(14) 71171-4$

Davies C et al (2013) Long-term effects of continuing adjuvant tamoxifen to 10 years versus stopping at 5 years after diagnosis of oestrogen receptor-positive breast cancer: ATLAS, a randomised trial. Lancet 381:805-816. https://doi.org/10.1016/s0140 $-6736(12) 61963-1$

Delozier T et al (2000) Delayed adjuvant tamoxifen: ten-year results of a collaborative randomized controlled trial in early breast cancer (TAM-02 trial). Ann Oncol 11:515-519

DeMichele A, Yee D, Esserman L (2017) Mechanisms of resistance to neoadjuvant chemotherapy in breast cancer. N Engl J Med 377:2287-2289. https://doi.org/10.1056/NEJMcibr1711545

Early Breast Cancer Trialists' Collaborative Group (1992) Systemic treatment of early breast cancer by hormonal, cytotoxic, or immune therapy. 133 randomised trials involving 31,000 recurrences and 24,000 deaths among 75,000 women. Early Breast Cancer Trial Collabor Group Lancet 339:1-15

Early Breast Cancer Trialists' Collaborative Group (1998) Tamoxifen for early breast cancer: an overview of the randomised trials. Early Breast Cancer Trial Collabor Group Lancet 351:1451-1467

Early Breast Cancer Trialists' Collaborative Group (2011) Relevance of breast cancer hormone receptors and other factors to the efficacy of adjuvant tamoxifen: patient-level meta-analysis of randomised trials. Lancet 378:771-784. https://doi.org/10.1016/ S0140-6736(11)60993-8

Early Breast Cancer Trialists' Collaborative Group (2015) Aromatase inhibitors versus tamoxifen in early breast cancer: patient-level meta-analysis of the randomised trials. Lancet 386:1341-1352. https://doi.org/10.1016/s0140-6736(15)61074-1

Ekholm M, Bendahl PO, Ferno M, Nordenskjold B, Stal O, Ryden L (2016) Two years of adjuvant tamoxifen provides a survival benefit compared with no systemic treatment in premenopausal patients with primary breast cancer: long-term follow-up $(>25$ years) of the Phase III SBII:2pre Trial. J Clin Oncol 34:22322238. https://doi.org/10.1200/jco.2015.65.6272

Engel J, Weichert W, Jung A, Emeny R, Holzel D (2019) Lymph node infiltration, parallel metastasis and treatment success in breast cancer. Breast (Edinburgh, Scotland) 48:1-6. https://doi. org/10.1016/j.breast.2019.07.008

Gagliato DDM et al (2014) Clinical impact of delaying initiation of adjuvant chemotherapy in patients with breast cancer. J Clin Oncol 32:735-744. https://doi.org/10.1200/jco.2013.49.7693

Gierach GL et al (2016) Association of adjuvant tamoxifen and aromatase inhibitor therapy with contralateral breast cancer risk among US women with breast cancer in a general community setting. JAMA Oncol. https://doi.org/10.1001/jamaoncol.2016.3340

Gnant M, Steger G, Greil R (2018) A prospective randomized multi-center phase-III trial of additional 2 versus additional 5 years of anastrozole after initial 5 years of adjuvant endocrine therapy-results from 3,484 postmenopausal women in the ABCSG-16 trial [abstract]. Cancer Res 78(4 Suppl):Abstract nr GS3-01

Goss PE et al (2016) Extending aromatase-inhibitor adjuvant therapy to 10 years. N Engl J Med 375:209-219. https://doi.org/10.1056/ nejmoa1604700

Gray RG (2013) Long-term effects of continuing adjuvant tamoxifen to 10 years versus stopping at 5 years in 6,953 women with early breast cancer. J Clin Oncol 31:5

Harbeck N et al (2019) Breast cancer. Nat Rev Dis Prim 5:66. https:// doi.org/10.1038/s41572-019-0111-2

Hölzel D, Eckel R, Emeny R, Engel J (2010) Distant metastases do not metastasize. Cancer metastasis reviews 29:737-750. https://doi. org/10.1007/s10555-010-9260-1

Hölzel D et al (2017) Improved systemic treatment for early breast cancer improves cure rates, modifies metastatic pattern and shortens post-metastatic survival: 35-year results from the Munich Cancer Registry. J Cancer Res Clin Oncol 143:1701-1712. https://doi. org/10.1007/s00432-017-2428-0

Jordan VC, Allen KE (1980) Evaluation of the antitumour activity of the non-steroidal antioestrogen monohydroxytamoxifen in the DMBA-induced rat mammary carcinoma model. Eur J Cancer (Oxford, England : 1990) 16:239-251

Mamounas EP et al (2019) Use of letrozole after aromatase inhibitorbased therapy in postmenopausal breast cancer (NRG Oncology/ NSABP B-42): a randomised, double-blind, placebo-controlled, phase 3 trial. Lancet Oncol 20:88-99. https://doi.org/10.1016/ s1470-2045(18)30621-1

Metcalfe K et al (2004) Contralateral breast cancer in BRCA1 and BRCA2 mutation carriers. J Clin Oncol 22:2328-2335. https:// doi.org/10.1200/jco.2004.04.033

Michailidou K et al (2017) Association analysis identifies 65 new breast cancer risk loci. Nature. https://doi.org/10.1038/nature24284

Munich Cancer Registry http://www.tumorregister-muenchen.de/en/. Accessed 25 May 2020

Noone A, Howlader N, Krapcho M, Miller D, Brest Ae SEER Cancer Statistics Review, 1975-2015 National Cancer Institute. Bethesda, MD. http://seer.cancer.gov/. Accessed 25 May 2020

Pan H et al (2017) 20-year risks of breast-cancer recurrence after stopping endocrine therapy at 5 years. N Engl J Med 377:1836-1846. https://doi.org/10.1056/nejmoa1701830

Rutqvist LE, Johansson H (2007) Long-term follow-up of the randomized Stockholm trial on adjuvant tamoxifen among postmenopausal patients with early stage breast cancer. Acta Oncol (Stockholm, Sweden) 46:133-145. https://doi.org/10.1080/0284186060 1034834

Smith IE, Yeo B, Schiavon G (2014) The optimal duration and selection of adjuvant endocrine therapy for breast cancer: how long is enough? Am Soc Clin Oncol Educ Book:e16-24. https://doi. org/10.14694/edbook_am.2014.34.e16

Swedish Breast Cancer Cooperative Group (1996) Randomized trial of two versus five years of adjuvant tamoxifen for postmenopausal early stage breast cancer. Swed Breast Cancer Cooper Group J Natl Cancer Inst 88:1543-1549

Veronesi A, Miolo G, Magri MD, Crivellari D, Scalone S, Bidoli E, Lombardi D (2010) Late tamoxifen in patients previously operated for breast cancer without postoperative tamoxifen: 5-year results of a single institution randomised study. BMC Cancer 10:205. https://doi.org/10.1186/1471-2407-10-205

Weedon-Fekjaer H, Lindqvist BH, Vatten LJ, Aalen OO, Tretli S (2008) Breast cancer tumor growth estimated through mammography screening data. BCR 10:R41. https://doi.org/10.1186/bcr2092

Welch HG, Prorok PC, O'Malley AJ, Kramer BS (2016) Breastcancer tumor size, overdiagnosis, and mammography 
screening effectiveness. N Engl J Med 375:1438-1447. https:// doi.org/10.1056/NEJMoa1600249

Yates LR et al (2017) Genomic evolution of breast cancer metastasis and relapse. Cancer Cell 32:169-184.e167. https://doi. org/10.1016/j.ccell.2017.07.005

Yung R et al (2020) The association of delay in curative intent treatment with survival among breast cancer patients: findings from the Women's Health Initiative. Breast Cancer Res Treat. https:// doi.org/10.1007/s10549-020-05572-y

Publisher's Note Springer Nature remains neutral with regard to jurisdictional claims in published maps and institutional affiliations. 